\title{
The WaveCat $\subset$ - Development of a new Wave Energy Converter
}

\author{
Gregorio Iglesias ${ }^{1, *}$, Hernán Fernández ${ }^{1}$, Rodrigo Carballo ${ }^{1}$, Alberte Castro ${ }^{1}$, Francisco \\ Taveira-Pinto $^{2}$ \\ ${ }^{1}$ University of Santiago de Compostela, Spain \\ ${ }^{2}$ Faculty of Engineering, University of Porto, Portugal \\ *Corresponding author. Tel: +34 982823650, Fax: +34 982285926, E-mail: gregorio.iglesias@usc.es
}

\begin{abstract}
The development of efficient, reliable Wave Energy Converters (WECs) is a fundamental prerequisite for wave energy to become a commercially viable energy source. Intensive research is currently under way on various technologies, among which WaveCat $\odot$ - a new WEC recently patented by the University of Santiago de Compostela. The purpose of this paper is to present the WaveCat concept and the ongoing work toward its development. WaveCat is a floating offshore WEC whose principle of operation is wave overtopping. It consists of two hulls, like a catamaran (hence its name). Unlike a catamaran, however, the hulls are not parallel but convergent-they are joined at the stern, forming a wedge in plan view. The methodology adopted to develop this patent is based on physical model tests which are described in the paper. A 1:30 model was tested in a wave tank under regular and irregular waves; waves and overtopping rates were measured, as were the model displacements - the latter using an advanced motion capture system. The data thus obtained will be used to validate a 3D numerical model currently under development, which in turn will be used to optimize the design of WaveCat for best performance.
\end{abstract}

Keywords: Wave energy converter, Overtopping, Physical modelling, Numerical modelling, CFD.

\section{Nomenclature}

$\alpha \quad$ wedge angle ........................................... [-]

$H \quad$ wave height (regular waves)..................... $m$

$T \quad$ wave period (regular waves).......................

$H_{s} \quad$ significant wave height (irreg. waves)..... m

$T_{p}$ peak wave period (irreg. waves) .................

\section{Introduction}

In order to reduce the emissions of greenhouse gases it is crucial to work along two lines. The first is to develop the already operational renewable energy sources, such as wind or photovoltaic energy. The second is to research and develop new energy sources [1]. Among these, marine renewable energy has a great potential for development in Europe. The European Science Foundation estimates that "by 2050 Europe could source up to $50 \%$ of its electricity needs from Marine Renewable Energy" [2]. Although Marine Renewable Energy comprises many different energy sources (offshore wind, wave energy, tidal currents, ocean currents, salinity gradient, thermal gradient and marine biomass), those with the highest potential are arguably wave energy, offshore wind and tidal energy. Two main issues must be resolved, however, for wave energy to become a fully established, commercially viable energy source. First, the wave resource along the coastline must be assessed; it presents significant spatial and temporal variations (e.g. [1, 3-4]), as is the case of other renewables. Second, efficient, reliable and low-impact Energy Converters (WECs) must be developed. This paper deals with WaveCat, a recently patented WEC. Its objectives are: (i) to present the WaveCat concept; and (ii) to describe the methodology used for its development, centred around physical model tests conducted in a 3D wave tank.

\section{The WaveCat concept}

WaveCat is a floating WEC intended for offshore deployment (water depths of 50-100 m), which has the advantage of a higher wave energy potential relative to onshore or nearshore 
locations (wave energy decreases as waves approach the shoreline). Another advantage of WaveCat is its low visual impact. The name WaveCat alludes to the fact that it is composed of two hulls, like a catamaran. Unlike a catamaran, however, these hulls are not parallel but convergent. The single-point mooring to a catenary-buoy allows the device to swing as the wave direction changes, thereby ensuring that the wedge opening always faces the waves (Figure 1). As waves propagate into the wedge, their height is enhanced by the convergence of the lateral boundaries (the hulls) until, eventually, they overtop the inner hull sides. Overtopping water is temporarily collected in on-deck tanks. The higher water level in these tanks relative to the sea level is taken advantage of to propel ultra-low head turbines as the water is drained back to sea. A fundamental issue in designing a WEC-especially an offshore WEC - is its survivability, i.e. its ability to sustain heavy storm conditions. The design of WaveCat includes a number of elements aimed at survivability, most notably the possibility of varying the angle formed by the hulls (hereafter referred to as wedge angle) between $120^{\circ}$ and $0^{\circ}$ according to the sea state. When a storm approaches, the angle is reduced to $0^{\circ}$, i.e. the wedge is closed, thereby transforming WaveCat into a monohullsimilar to a conventional ship from the standpoint of seakeeping. Freeboard and draft are also variable: in the model they were varied by means of solid ballast; in the prototype, ballast tanks filled with water will be used (as in ships). The prototype hull length is $90 \mathrm{~m}$.
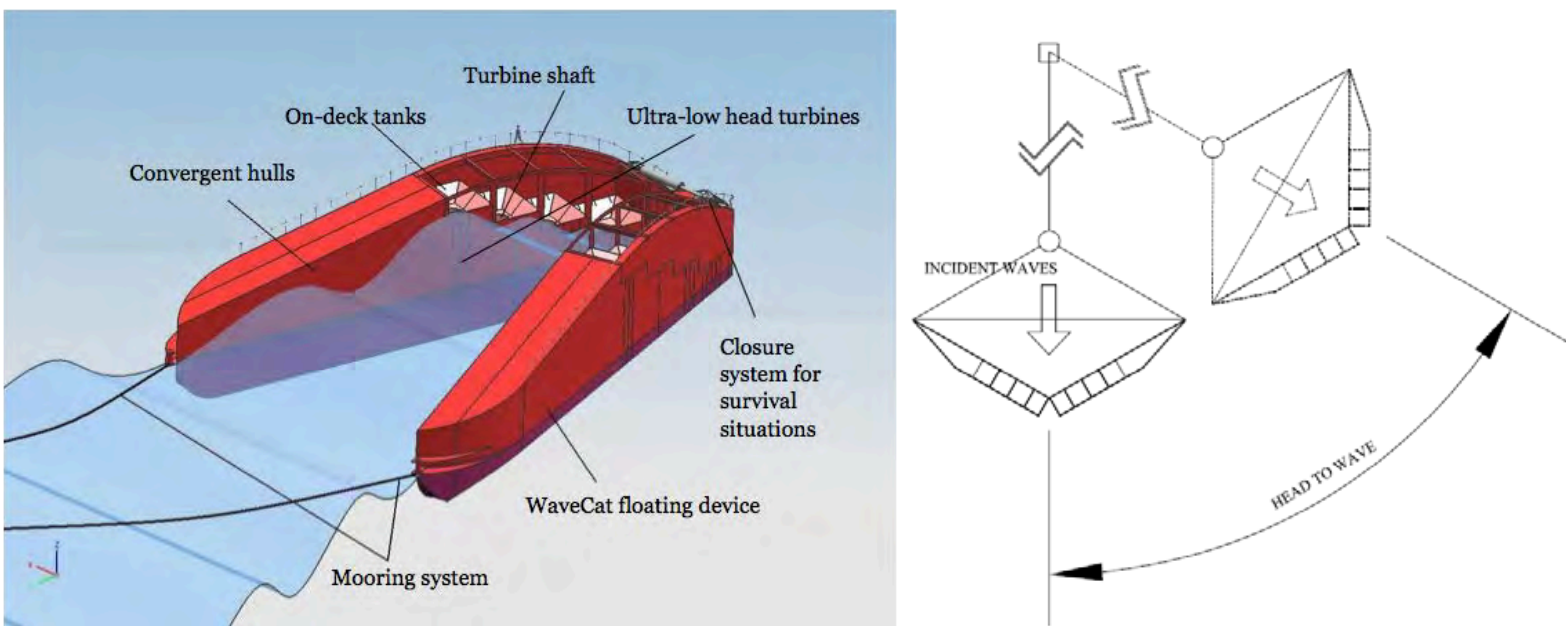

Fig. 1. The WaveCat concept (left) and a plan view of the single-point mooring system (right).

\section{Methodology}

The research and development of WaveCat combines physical and numerical modeling. So far, physical model tests in a wave tank have been completed. The methodology of these tests is the focus of this section (subsections 3.1 to 3.3). The numerical model, which is currently under development, is briefly presented in subsection 3.4.

\subsection{Physical model}

The 3D model, constructed of marine board, represented the WaveCat at a 1:30 scale (Figure 2). Tests were conducted at the wave tank of the University of Porto, with dimensions of $28 \times$ $12 \times 1.25 \mathrm{~m}$; at its centre was a pit with dimensions of $4.5 \times 2 \times 1.5 \mathrm{~m}$ (Figure 3). The catenary-buoy mooring system was anchored at the front end of the pit (Figure 4). Wave generation was carried out with a directional (multielement) piston-type wavemaker. The experimental setup included six wave gauges aligned with the centreline of the tank, and four other on the model (one for each water tank). The quiescent water depth in the tank was set to $0.90 \mathrm{~m}$ (or $2.40 \mathrm{~m}$ in the central pit) for all tests. 


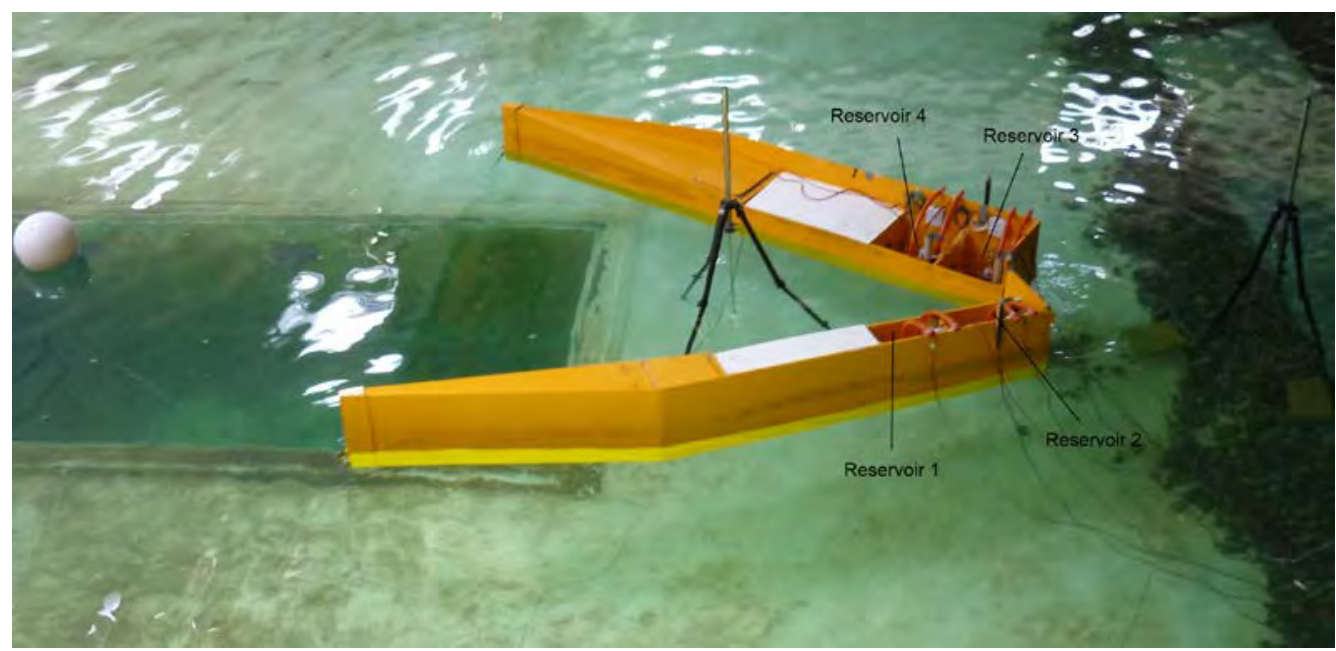

Fig. 2. The physical model in the wave tank with its four reservoirs for collecting overtopping water.

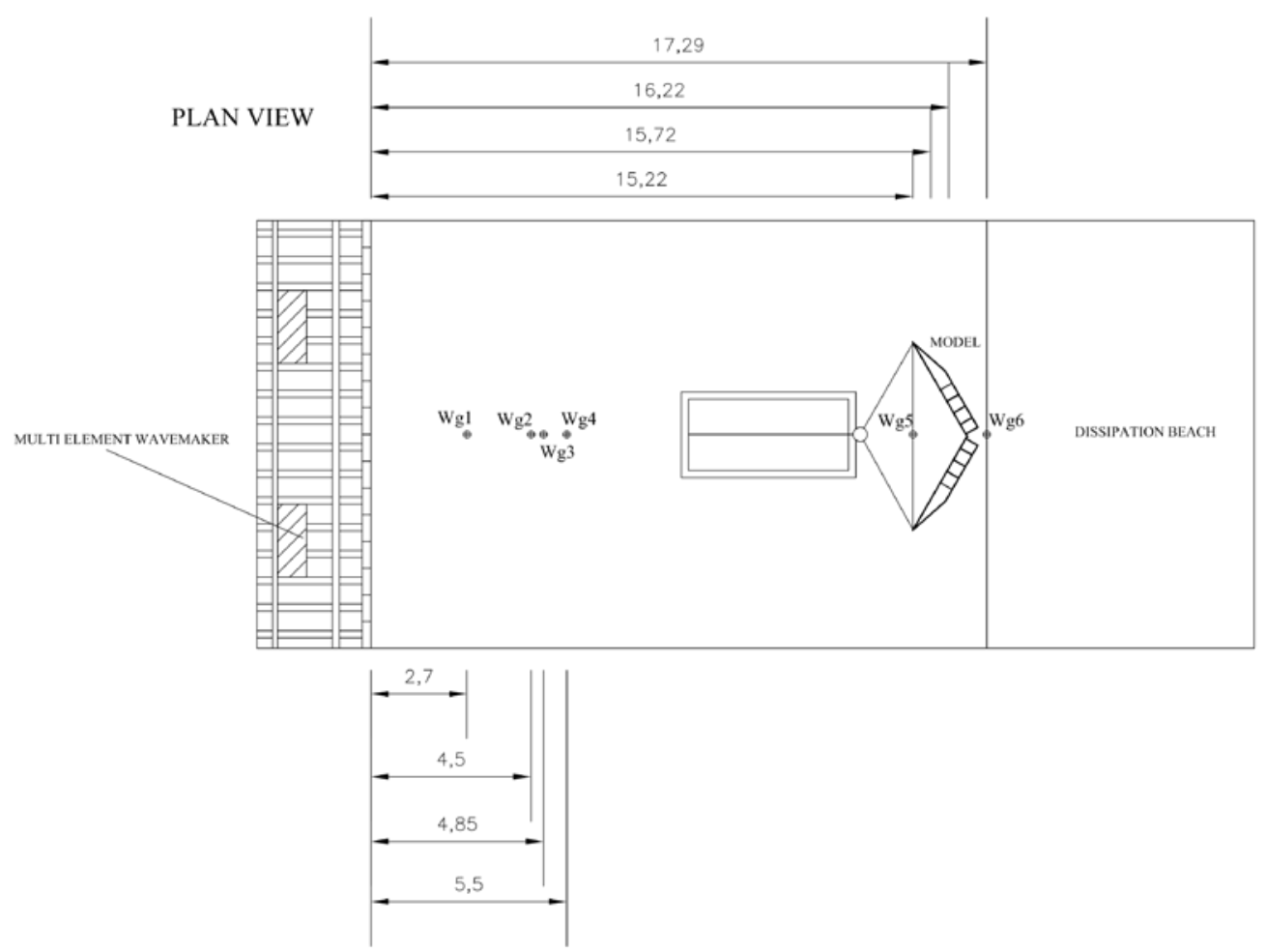

Fig. 3. Wave tank layout and experimental setup, showing the WaveCat model and the location of the wave gauges outside the model (Wg1 to Wg6). [Dimensions in $\mathrm{m}$ ].

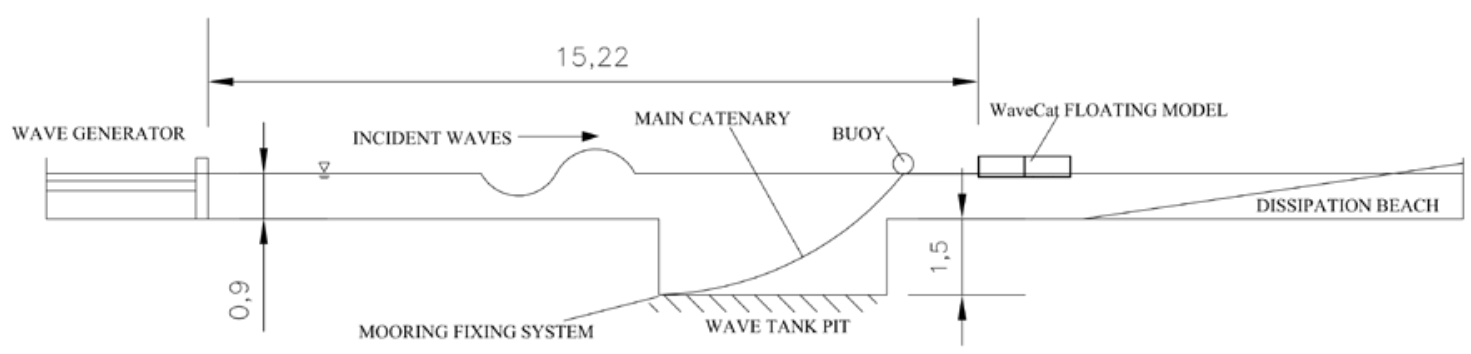

Fig. 4. Longitudinal section of the wave tank showing the model and the catenary-buoy mooring system. [Dimensions in $m$ ]. 


\subsection{Experimental campaign}

In total, the experimental campaign comprised 43 tests, 25 of which with regular waves and 18 with irregular waves. The wedge angle $(\alpha)$ was varied between four values: $30^{\circ}, 45^{\circ}, 60^{\circ}$ and $90^{\circ}$. Three quiescent freeboard values $\left(F_{b}\right)$ were used: $0.04 \mathrm{~m}, 0.09 \mathrm{~m}$ and $0.10 \mathrm{~m}$. Regular waves were in the ranges $H=0.07-0.10 \mathrm{~m}$ and $T=1.65-2.20 \mathrm{~s}$. Irregular waves varied in the ranges $H_{s}=0.067-0.100 \mathrm{~m}$ and $T_{p}=1.83-2.20 \mathrm{~s}$. For illustration the parameters in some of the tests (the irregular wave tests with the lowest freeboard) are shown in Table 1.

Table 1. Parameters in the irregular wave tests with a quiescent freeboard $F_{b}=0.04 \mathrm{~m}$.

\begin{tabular}{cccc}
\hline Test case & $\alpha\left(^{\circ}\right)$ & $H_{s}(\mathrm{~m})$ & $T_{p}(\mathrm{~s})$ \\
\hline AA07_I3 & 60 & 0.083 & 2.013 \\
AA07_I5 & 60 & 0.100 & 2.196 \\
AB07_I3 & 90 & 0.083 & 2.013 \\
AB07_I5 & 90 & 0.100 & 2.196 \\
AD07_I3 & 45 & 0.083 & 2.013 \\
AD07_I5 & 45 & 0.100 & 2.196 \\
AE07_I3 & 30 & 0.083 & 2.013 \\
AE07_I5 & 30 & 0.100 & 2.196 \\
\hline
\end{tabular}

Each of the four water reservoirs (two per hull) in the WaveCat model is equipped with a pump and a control system (Figure 5). The control system operates based on the water level in the reservoir as measured by a capacitance-type gauge. The pump begins to function when the water reaches a certain (maximum) level, and stops when it has gone down to a minimum value. The water level in two reservoirs, \#3 and 4, during the test AD07_I5 is shown in Figure 6 ; the intervals of pump operation correspond to the near vertical lines of the graph. A typical record of the free surface level during the same test is shown in Figure 7.

In the model, for simplicity, the pumps worked with a constant flowrate, and during (generally short) intervals of time. It is important to mention that this pumping system in the model is not intended to replicate the functioning of the turbines in the prototype, but merely to allow a longer test duration. Instead, the control system in the prototype will aim for continuous operation of the turbines, with the tanks acting as buffers to provide continuous outflow toward the turbines in spite of the discontinuous nature of the overtopping events. The outflow rate in the prototype will be set by its own control system so as to maintain the continuous turbine operation, taking into account the overtopping rate (which depends on the sea state) and the turbine-generator characteristics.

\subsection{Measurement of model displacements}

During the tests, the displacements of the model under wave action were recorded by means of a motion capture system consisting of three infrared video cameras, reflective elements on the model, a dedicated computer and ad hoc software. The cameras detected the positions of a number of small reflective spheres installed at different points on the model (Figure 8). Their motions were then converted by the system software into model displacements along the three coordinate axis (heave, surge, sway) and rotations around them (roll, pitch, yaw). For illustration, the pitch and roll of the model during test AD07_I5 are shown in Figure 9. 


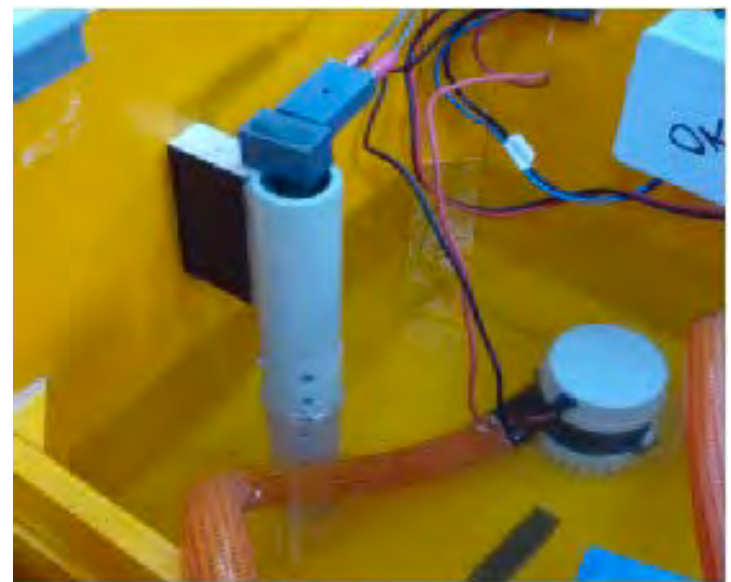

Fig. 5. Pump and control system in one of the water reservoirs of the model.
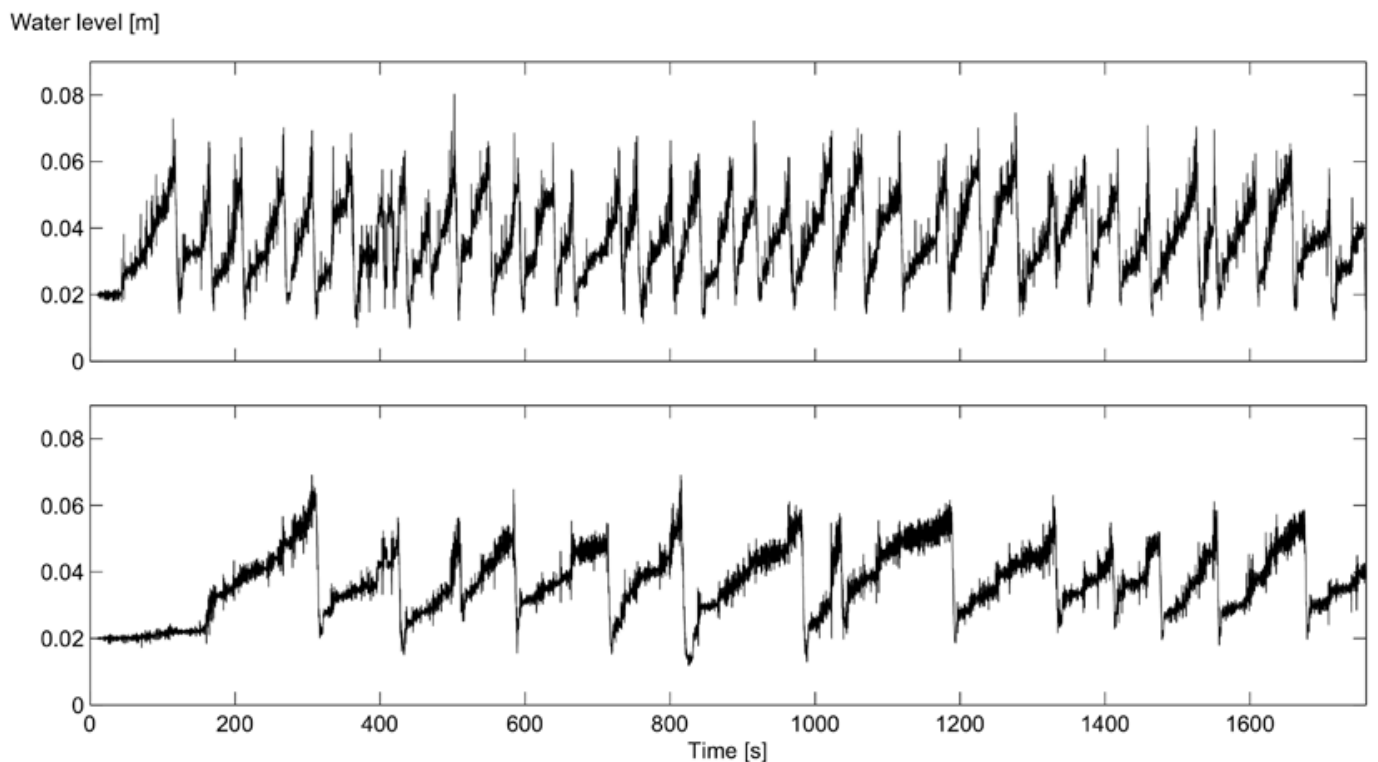

Fig. 6. Water level in reservoirs \#3 (aft reservoir, above) and \#4 (fore reservoir, below) during test AD07_I5. [Refer to Figure 3 for the location of the reservoirs in the model].

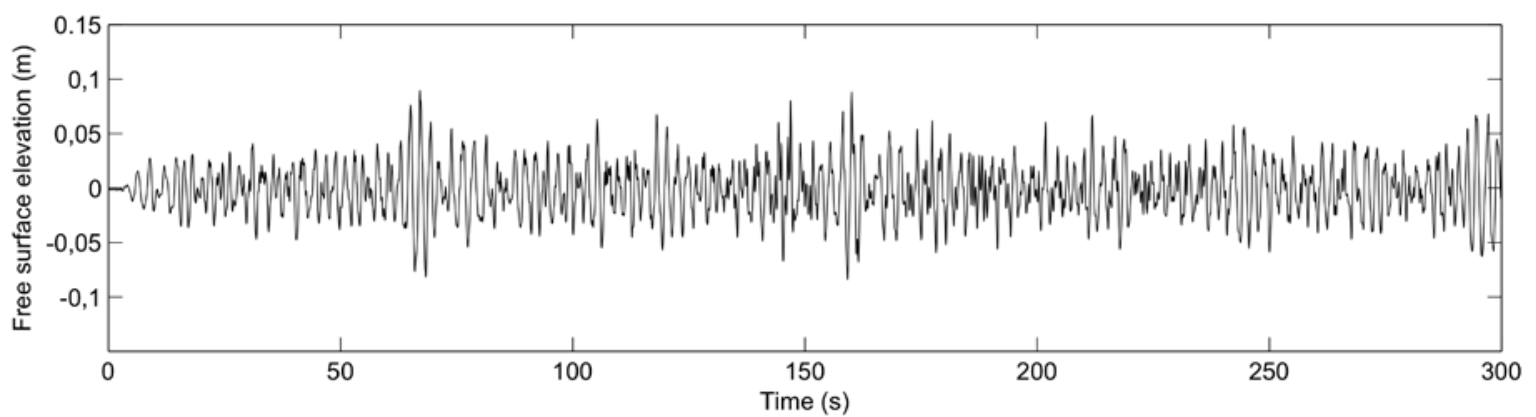

Fig. 7. Free surface elevation signal at the wave gauge in front of the model (Wg4) during test AD07_I5. [For clarity, only the first 5 min of the test are shown].

\subsection{Numerical model}

The development of a numerical model for WaveCat started with a 2D RANS-VOF model. This model was successfully validated using results from 2D physical model tests carried out 
in the wave flume of the University of Santiago de Compostela. Currently a 3D numerical model is being implemented; the model solves the RANS (Reynolds-Averaged NavierStokes) equations with a volume-of-fluid approach, using a state-of-the-art parallel code (Star-CCM+). The model simulates the WaveCat response as a floating body interacting with waves. It is expected that this model will be validated in the (hopefully near) future based on the results of the physical model tests presented above. A preliminary image from a simulation is shown in Figure 10.

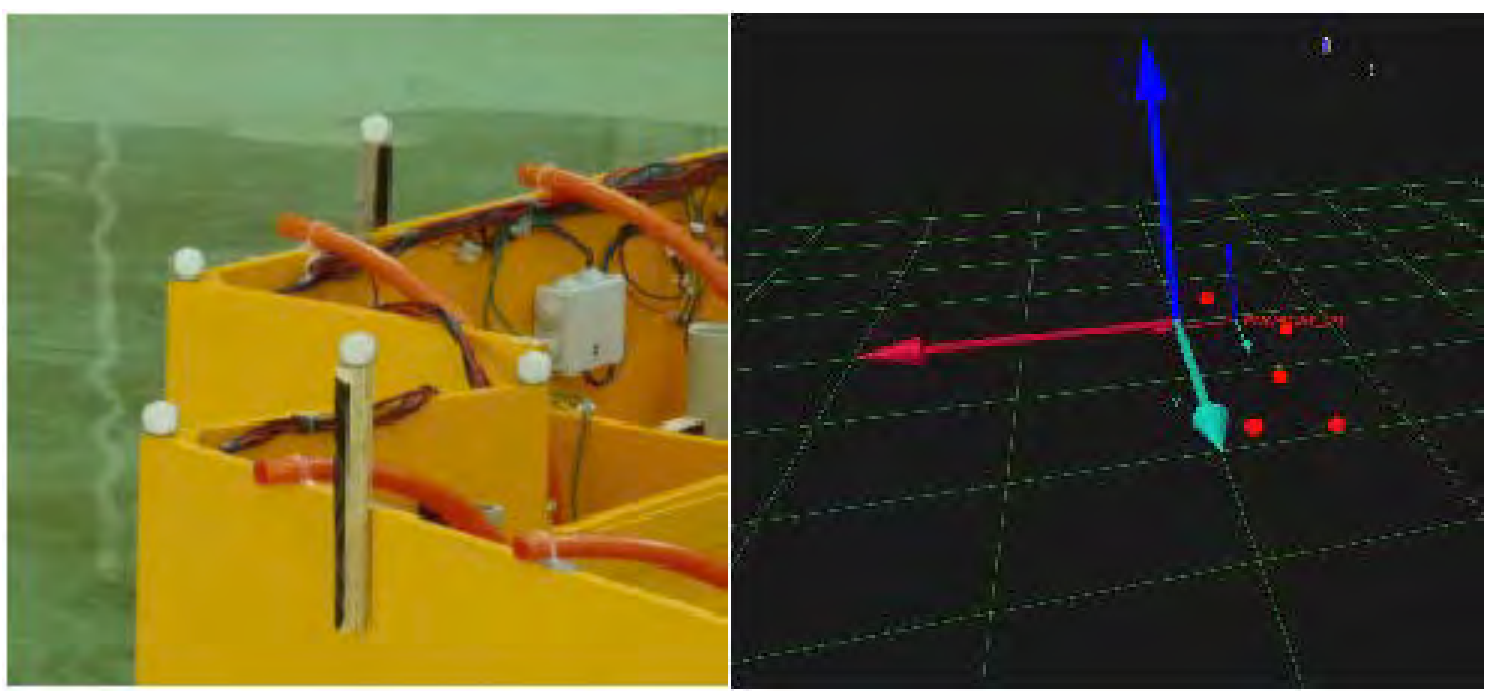

Fig. 8. White reflective spheres on the model (left) and detected by the motion capture system (right).
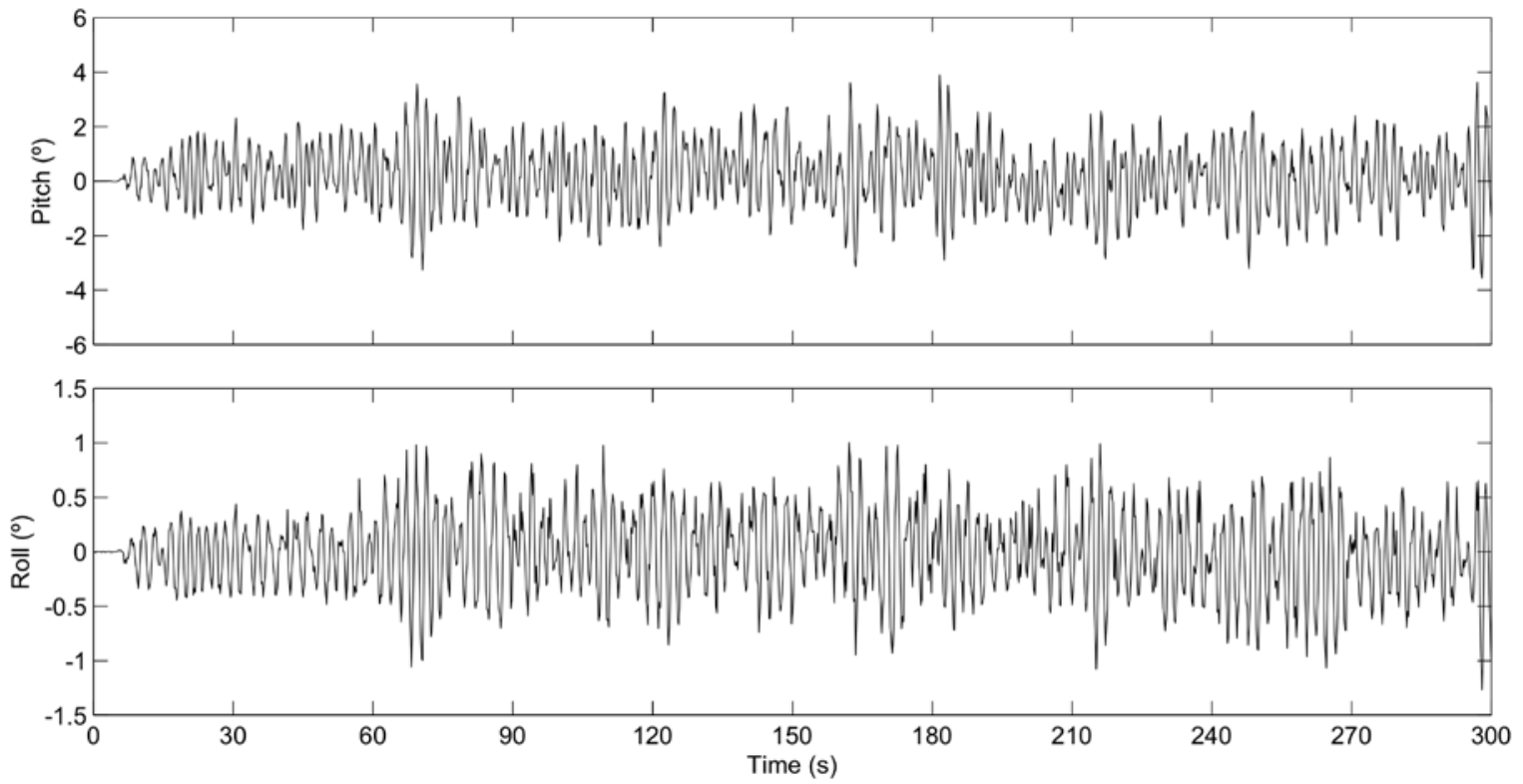

Fig. 9. Pitch and roll during test AD07_I5. [For clarity, only the first 5 min of the test are shown]. 


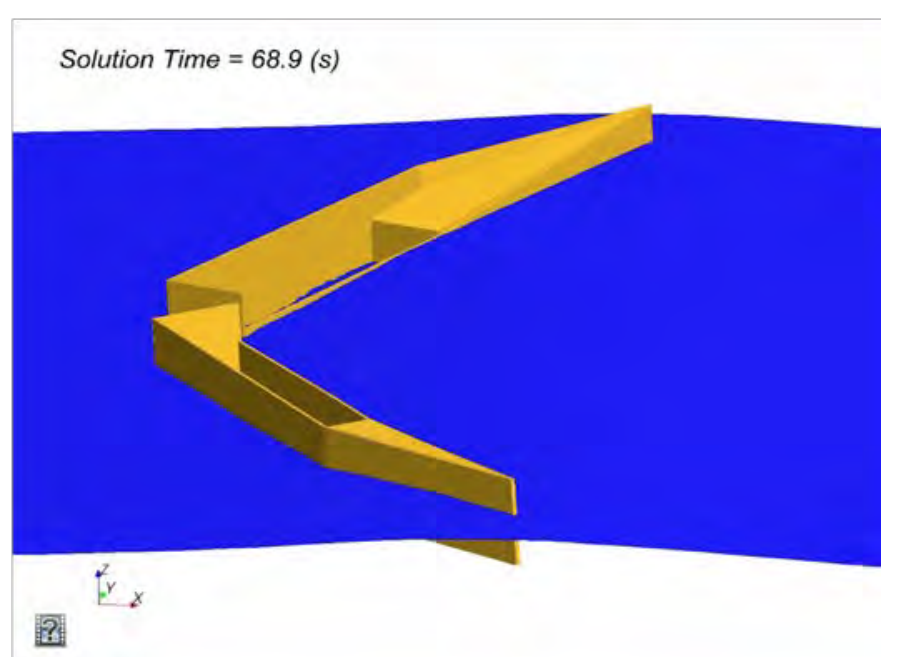

Fig. 10. One of the video frames of a simulation with the 3D numerical model under development.

\section{Results and Discussion}

The present paper presents the WaveCat, a recently patented WEC, and the ongoing work to develop it as a commercially viable system. A 1:30 model was constructed and tested in a large wave tank. In total 43 tests were carried out, both with regular and irregular waves. In addition to the wave parameters (wave height and period in the case of regular waves, significant wave height and peak period in the case of irregular waves), two fundamental model parameters were varied in the tests: wedge angle and freeboard. The motions of the model during the tests were measured by means of a motion capture system which included three infrared video cameras.

The results of the tests may be classified into three different levels or categories. First, on a conceptual level, the tests enabled to verify the WaveCat as a valid concept for wave energy conversion. The second level concerns the design of WaveCat and, in particular, of the water reservoirs. In the tested model the two reservoirs in each hull have the same volume and occupy the same length along the hull side. In Figure 6 it is apparent that the aft reservoir (\#3) experiences significantly heavier overtopping than the fore reservoir (\#4) during test AD07_I5. This imbalance was consistently observed throughout the experimental campaign, the aft reservoir collecting larger volumes of water than the fore reservoir. If the turbinegenerator configuration in the prototype is the same for both reservoirs, this consistent difference in overtopping rates is clearly suboptimal. One method to overcome this problem is to increase the volume of the fore reservoir by extending its length along the hull side at the expense of the aft reservoir, to the extent necessary to balance the overtopping rates. The other is to maintain the same volume and length along the hull side for both reservoirs, as in the tested model, but to use different turbine-generator configurations in the prototype-the aft reservoir would have a turbine-generator with greater rated power than the fore reservoir, in accordance with its larger overtopping rate. Although the first option would appear to be more attractive, no definitive decision has been taken so far. Finally, the third level of results comprises the time series of overtopping rates and model displacements and rotations gathered during the tests, which will be used to validate the 3D numerical model currently under development. Once validated, the model will be used to optimize the design of WaveCat for best efficiency under a given set of wave conditions, which will be chosen according to the wave climate of the deployment area. Thus, the physical model tests presented in this paper are a crucial step in the development process of this new WEC. 
The WaveCat concept (Section 2) presents four main advantages with respect to other WECs. In the first place, its design with two converging hulls and, in particular, the fact that the angle between them can be varied according to the sea state constitute a significant asset for survivability; in effect, under extreme (storm) conditions the wedge can be closed, thereby transforming WaveCat into a monohull vessel. In the full size WaveCat, a "locking system" will be provided to keep both hulls together during a storm without creating excessive stresses on the bow hinge. The freeboard on the outer hull sides is considerably larger than that on the inner hull sides. With no waves overtopping the inner hull sides, the survivability of WaveCat is greatly enhanced. The second advantage of the WaveCat design is that the wedge angle can be varied during normal operation to optimize the efficiency - the smaller the waves, the larger the wedge angle. Third, the moving parts activated by the waves are only the turbinegenerators; there are no complex joints moving with the passage of each wave, as is the case of other WECs. This may be expected to result in better reliability-and reliability is a key aspect of economic viability. Finally, the water tanks are placed along the hulls, rather than at the back of the wedge; therefore, the motions of WaveCat in waves may be expected to affect the overtopping rate less, merely causing a displacement along the hull of the point where overtopping begins. If the wave tanks along the hulls were substituted by a single tank at the back of the wedge, the motions of the WEC-in particular, its heave-could significantly reduce the overtopping rates under resonant conditions.

\section{Conclusions}

The development of a new Wave Energy Converter is a long process. At the current stage of development of WaveCat, wave tank tests of a 1:30 model were successfully completed, with an experimental setup that included an advanced motion capture system. With these physical model tests, involving many different sea states and model configurations (different wedge angles and freeboards), the WaveCat concept as a wave energy conversion system was verified. A second conclusion refers to the design of the water reservoirs. In view of the consistent imbalance in overtopping rates that was found during the experimental campaign there are two main options. Either the design of the tested model is kept in the prototype, in which case the turbine-generator configuration in the aft reservoirs must be different from that in the fore reservoirs (with greater rated power in the aft reservoirs), or the volume of the fore reservoir is increased at the expense of the aft reservoir, in which case the same turbinegenerator configuration can then be used for both reservoirs. Finally, the data on model motions (displacements and rotations) and overtopping rates obtained in the experimental campaign presented in this paper will be the basis on which the 3D numerical model currently under development will be validated. This model will enable to optimize the design of WaveCat for best performance under specified wave conditions. Upon optimization, the next step will be the construction of a full-size demonstrator and its sea trial.

\section{References}

[1] G. Iglesias, M. López, R. Carballo, A. Castro, J.A. Fraguela, P. Frigaard, Wave energy potential in Galicia (NW Spain), Renewable Energy 34, 2009, pp. 2323-2333.

[2] European Science Foundation, Marine Board Vision Document 2, 2010.

[3] G. Iglesias, R. Carballo, Wave energy potential along the Death Coast (Spain), Energy 34, 2009, pp. 1963-1975.

[4] G. Iglesias, R. Carballo, Wave energy resource in the Estaca de Bares area (Spain), Renewable Energy 35, 2010, pp. 1574-1584. 